\section{Mimetización de corona cerámica libre de metal de un incisivo central superior. Reporte de caso}

\section{Mimicry of metal-free ceramic crown of an upper central incisor. Case report}

\section{Resumen}

Actualmente, la estética dental presenta un papel relevante dentro de las relaciones interpersonales y la autoestima de los pacientes, es por eso que la demanda de tratamientos estéticos y conservadores son cada vez más frecuentes en la consulta dental, esta demanda compromete a los profesionales a una mayor preparación para identificar los procedimientos más adecuados a ejecutarse. Para el odontólogo es un reto conseguir una completa armonización e integración de los tratamientos efectuados en el sector anterior y más aún cuando se requiere reemplazar un único elemento dentario. El presente reporte de caso tiene por objetivo describir un caso de rehabilitación de alta complejidad de un incisivo central superior fracturado, cuyas características de color y forma fueron recreadas a través de un correcto intercambio de información entre el odontólogo y el técnico dental, obteniendo una corona de disilicato de litio con una excelente mimetización.

Palabras clave: Coronas dentales; Cerámicas; Estética Dental; Rehabilitación bucal (fuente: DeCS BIREME).

\begin{abstract}
Nowadays, dental aesthetics has a relevant role within interpersonal relationships and the self-esteem of patients, that is the reason the demand for aesthetic and conservative treatments are more frequent in dental practice, this demand commits professionals to a greater preparation, in order to identify the most appropriate procedures to be executed. For the dentist it is a challenge to achieve a complete harmonization and integration of the treatments carried out in the anterior sector and even more when it is necessary to replace a single dental element. The objective of this case report is to describe a highly complex rehabilitation case of a fractured upper central incisor, whose color and shape characteristics were recreated through a correct exchange of information between the dentist and the dental technician, obtaining a lithium disilicate crown with excellent mimicry.
\end{abstract}

Keywords: Crowns; Ceramics; Dental esthetics; Mouth rehabilitation (source: MeSH NLM).

\section{Caso Clínico}

Boris Lenin Ochoa-Vázquez ${ }^{1, a}$, Sara Ivanna

Cedillo-Orellana ${ }^{2, b}$, Rommel Patricio

Guachizaca-Uyaguari 1,c, Diana Karolina Gallegos-Torres 1,0

${ }^{1}$ Práctica privada.

2 Universidad Católica de Cuenca, Cuenca, Ecuador.

a Especialista en Rehabilitación Oral e Implantología.

${ }^{\circ}$ Especialista en Endodoncia.

${ }^{\circ}$ Cirujano Dentista.

\section{Correspondencia:}

Boris Lenin Ochoa-Vázquez: borisov23@hotmail.com Alfonso Moreno Mora 2-179 y Juan Î́niguez, Cuenca, Ecuador.

ORCID: 0000-0002-4092-0640

Coautores:

Sara Ivanna Cedillo-Orellana: ivannac90@hotmail.com ORCID: 0000-0001-9621-2048

Rommel Patricio Guachizaca-Uyaguari: patogua1105@ hotmail.com

ORCID: 0000-0001-6846-7131

Diana Karolina Gallegos-Torres: dkgt1993@hotmail.com ORCID: 0000-0002-2927-916X

\section{Editora:}

Rosse Mary Falcón-Antenucci

Universidad Inca Garcilaso de la Vega, Perú.

Conflicto de intereses: los autores declaran no tener conflictos de interés.

Fuente de financiamiento: autofinanciado.

Recibido: 29/04/21

Aceptado: 30/05/21

Publicado: 01/07/21 


\section{Introducción}

Los órganos dentarios debido a la edad, adoptan ciertas particularidades y detalles que modifican sus aspectos morfológicos, texturas y color convirtiéndose en un desafío a la hora de rehabilitar un diente aislado en el sector estético ${ }^{1}$. El odontólogo debe reconocer estas características biológicas, morfológicas, mecánicas y estéticas que hacen singular a cada diente; mediante un análisis individual y la correcta selección y manipulación del material, para luego ser replicadas en restauraciones completamente miméticas. En la percepción del color influyen tres factores: observador, fuente luminosa y el objetivo $^{2}$, la selección visual del color se considera una medición subjetiva, en relación al observador, ciertos factores pueden alterar esta medición, tales como: fatiga de la visión, edad, experiencia y deficiencia visual que pueden alterar los resultados ${ }^{3}$.

La técnica habitual para registrar las características ópticas de los dientes emplea guías o escalas de color que representan las tonalidades de los materiales disponibles con las cuales el odontólogo formará el mapa cromático, dividiendo al diente par o al más cercano a ser restaurado en tres secciones (cervical, medio e incisal), comparando cada sección con la guía para asignar el valor de color adecuado. El mapa cromático reconocerá y definirá matices, manchas, defectos e hipoplasias; se recomienda limpiar el diente de toda adherencia que disminuya la apreciación del color y textura, observar el diente bajo una buena iluminación junto a la guía por periodos de 15 segundos, el diente debe estar hidratado puesto que si no, aparecerá más claro y blanquecino, es conveniente que el clínico descanse la vista entre observaciones fijándola sobre una superficie de color suave, preferiblemente azul claro y finalmente dibujar los datos que se aprecien ${ }^{2,3}$.

Hoy en día se cuenta con instrumentos más sofisticados que facilitan la medición y reproductibilidad del color como: las cámaras digitales con sistemas de imagen, colorímetros, y espectrofotómetros, estos últimos con una precisión que alcanza el 93,3\% ${ }^{4}$. La fotografía dental se ha convertido en un recurso de gran valor a la hora de registrar y transferir información al laboratorio, no solo visualiza la morfología dental y colores, sino también texturas superficiales, distribuciones de color y condiciones intraorales, mejorando la caracterización de las restauraciones, además las fotografías en blanco y negro ayudan mucho al momento de definir el valor, facilitando la elección del material restaurador ${ }^{4,5}$.

Adicionalmente, la correcta comunicación entre el dentista y el técnico dental harán posible un resultado final de mejor calidad, menor tiempo de confección, menores errores y mayor fuente de conocimiento para ambas partes. El odontólogo debe transmitir la mayor cantidad de datos, en un lenguaje claro y conciso ya sea por simples notas en papel o usando medios tecnológicos a disposición; la relación laboratorio-dentista se ve fortalecido por el paso del tiempo, en el que cada uno desarrolla una técnica particular de trabajo ${ }^{6,7}$.
Antes las restauraciones de metal-cerámica cumplían buenas propiedades funcionales, sin embargo, la corrosión de las aleaciones, producto de la acción de la saliva, produce iones nocivos que son descargados en el medio bucal, hecho que no ocurre con las cerámicas por su baja reactividad química ${ }^{8}$. La búsqueda de nuevos materiales ha llevado al desarrollo y evolución de las cerámicas dentales, siendo capaces de emular propiedades como: el coeficiente de expansión térmica semejante al diente, biocompatibilidad biológica, alta resistencia a la compresión, resistencia a la abrasión, módulo de elasticidad similar a la dentina, traslucidez, estabilidad del color y resistencia al desgaste, soportando muy bien las cargas masticatorias al sustrato dentario. Así, las restauraciones de disilicato de litio presentan propiedades mecánicas que alcanzan los 400 a $440 \mathrm{MPa}$ con una alta tasa de supervivencia ( $92,7 \%$ a 3 años) ${ }^{9-11}$.

El objetivo de este reporte de caso clínico es exponer los procesos de planificación y comunicación clínico-laboratorial, para obtener una mimetización de alto nivel en una corona cerámica libre de metal.

\section{Reporte del caso}

Anamnesis. Paciente de sexo masculino de 69 años de edad, acude a consulta odontológica por presentar fractura de una prótesis fija de la pieza dental 1.1 (Figura 1), en el interrogatorio el paciente relata que la prótesis fija fue colocada hace 10 años. Además, refiere ser fumador y mantener un alto consumo de cafeína. El paciente solicita que su tratamiento se puntualice en la pieza fracturada.

Examen clínico. Al examen clínico se observó el remanente dentario de la pieza 1.1 y se determinó la existencia de $1,5 \mathrm{~mm}$ de tejido dental sano en sentido vertical y horizontal sobre el margen gingival proporcionando un correcto efecto ferrule, además se verificó que el conducto radicular se encontraba sellado con material resinoso, radiográficamente se observó la presencia de un tratamiento endodóntico previo en buenas condiciones $y$ tejidos periapicales sanos (Figura 2).

Diagnóstico. Pieza dental 1.1 con diagnóstico de fractura coronaria, tratamiento endodóntico previo y tejidos periapicales sanos.

Plan de tratamiento. En base a lo anterior, el plan de tratamiento aceptado por el paciente fue la rehabilitación de la pieza dental fracturada mediante una prótesis fija unitaria de disilicato de litio.

Ejecución del tratamiento. Previo a la ejecución del tratamiento el paciente firmó un consentimiento informado, en el cuál se indicó que para el manejo del caso se tomarán fotografías del proceso, y éstas serán divulgadas con fines académicos.

Primera consulta: se realizó la toma de impresión preliminar con alginato (Hydrogum, Zhermack, Badia Polesine, Italia) para la confección de un modelo de estudio, encerado de diagnóstico y obtención de llaves de silicona para la posterior provisionalización. 


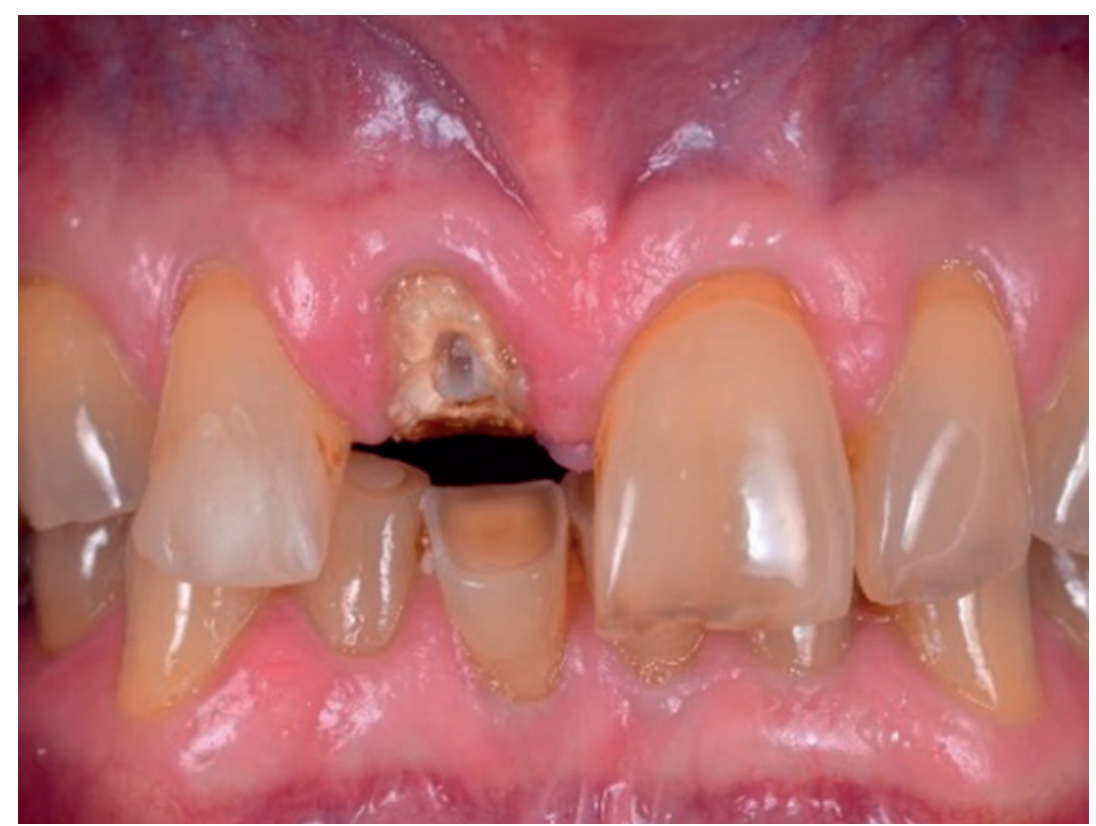

Figura 1. Aspecto clínico inicial

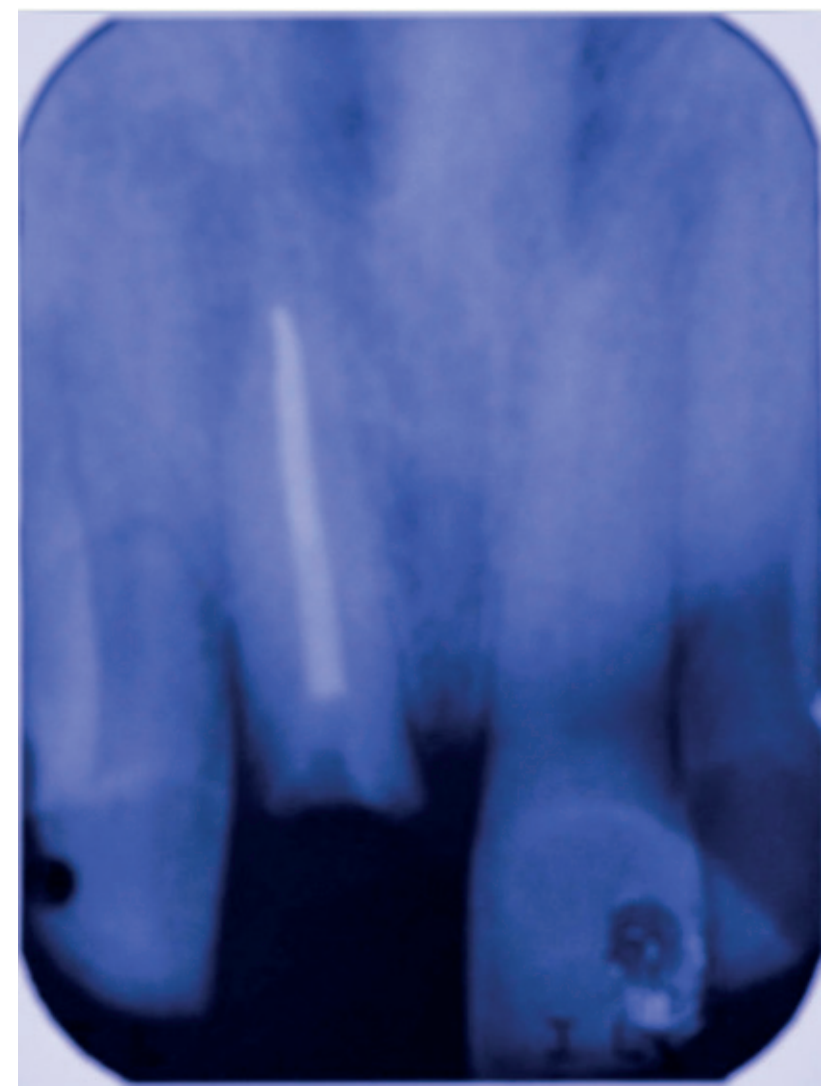

Figura 2. Radiografía periapical pieza 1.1

Segunda consulta: se realizó la desobturación del conducto radicular dejando $4 \mathrm{~mm}$ de sellado apical ${ }^{12,13}$, se cementó un poste de fibra de vidrio \#1 (WhitePost DC, FGM, Joinville, Brasil) con un sistema resinoso dual (ParaCore, Coltene, Alstätten, Suiza), reconstrucción de muñón con resina color B1 (Filtek P60, 3M, Minnesota, USA), preparación dental para corona cerámica libre de metal (incisal $2 \mathrm{~mm}$, distal y mesial $1 \mathrm{~mm}$, vestibular y palatino $1,5 \mathrm{~mm}$ ) (Figura 3) y confección de provisorio acrílico en color A3 (Alike, GC America Inc., Illinois, USA) utilizando llave de silicona previamente confeccionada (Figura 4).

Tercera consulta: al cabo de 8 días, se retiró la corona provisoria, limpiando la zona para determinar el estado de salud de los tejidos pericoronarios y se procedió a la toma de la impresión funcional en dos tiempos, empleando la técnica de doble hilo \#00 y \#0 (Ultrapack, 


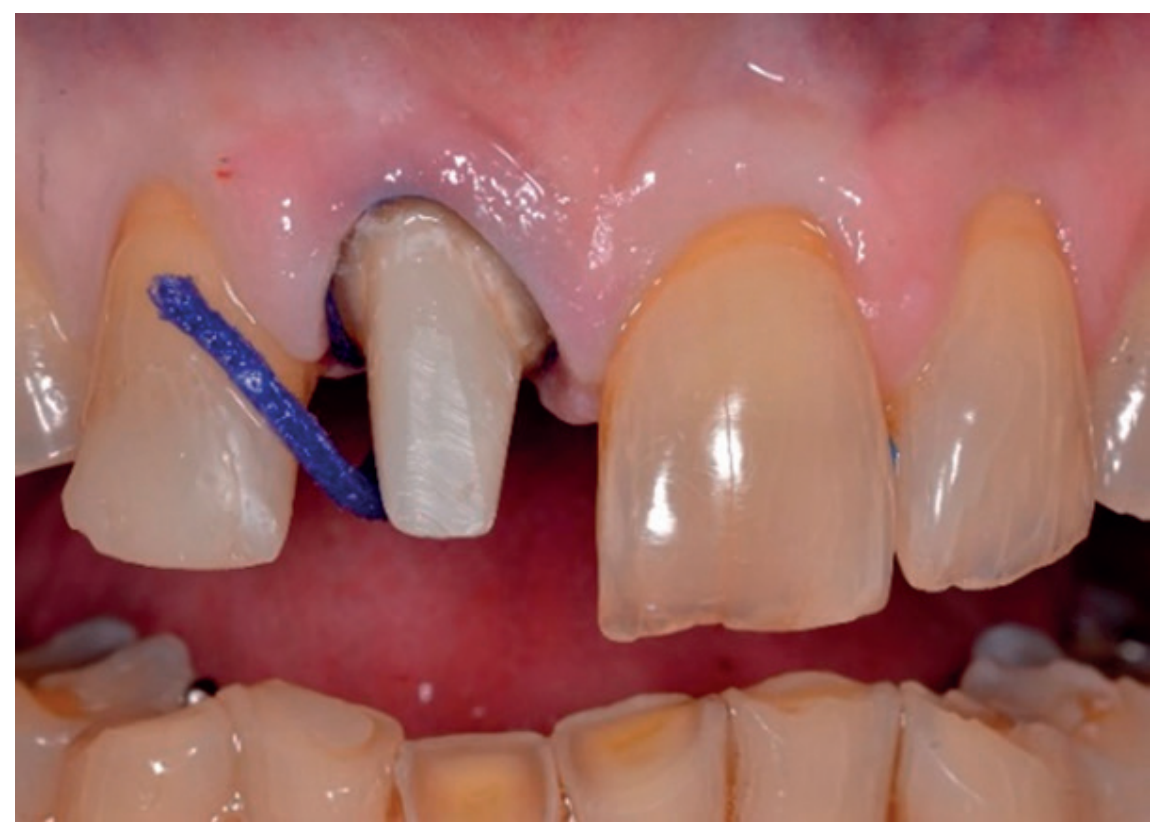

Figura 3. Preparación del muñón

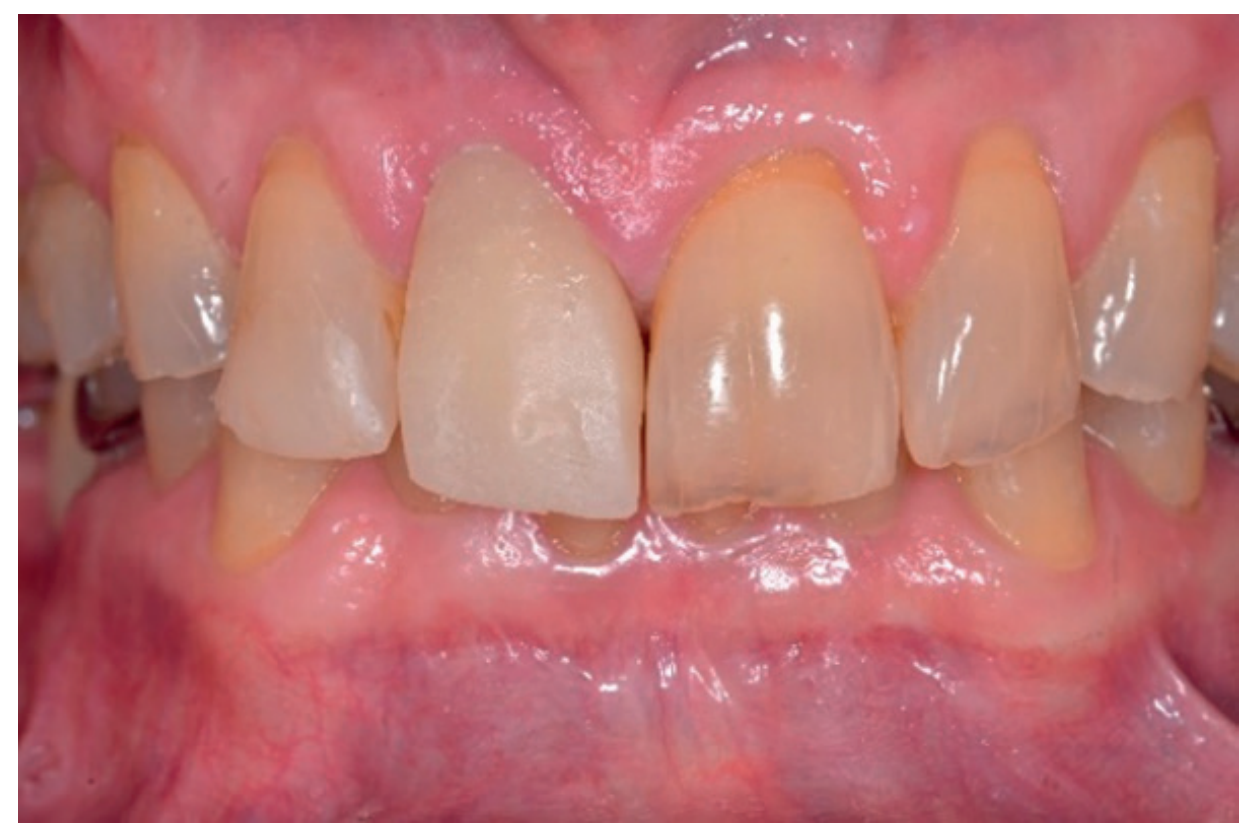

Figura 4. Corona provisional de acrílico

Ultradent, Utah, USA) con silicona por adición (Silagum, DGM, Hamburgo, Alemania) de consistencia pesada y liviana. El registro de color se realizó con la escala de color (Linearguide 3D-MASTER, Vita Zahnfabrik, Bad Säckingen, Alemania) comparando la guía con el diente homólogo, eligiendo el color $5 \mathrm{~m} 1$.

Para una mejor comunicación con el laboratorio se realizó el registro fotográfico a color (Figura 5A) para posterior mapeo y mejor apreciación de detalles, y en escala de grises (Figura 5B). para determinar el valor. Las fotografías del diente homólogo fueron tomadas en primer plano y con diferentes angulaciones con la finalidad de captar la mayor cantidad de detalles. Se usó una cámara fotográfica (D7000, Nikon, Tokyo, Japón) con un lente macro (100mm, Tokina), empleando twin flash (Speedlight R1C1, Nikon, Tokyo, Japón), más difusores de luz (Quik Bounce, Lumiquest, New Braunfels USA); para la programación de la cámara se empleó ISO 100, f25, velocidad $1 / 125$, WB, predeterminado para twin flash, potencia de flash $1 / 4$.

Se envió al laboratorio las fotografías junto con la orden de trabajo en donde constaba información como edad y género del paciente, color del muñón y material seleccionado; junto con esto se solicita al laboratorio el envío del mapa cromático y la guía de caracterizaciones previo a la confección de la corona.

Una vez aprobado el diseño (Figura 6), el laboratorio confeccionó la corona en disilicato de litio, pastillas IPS 


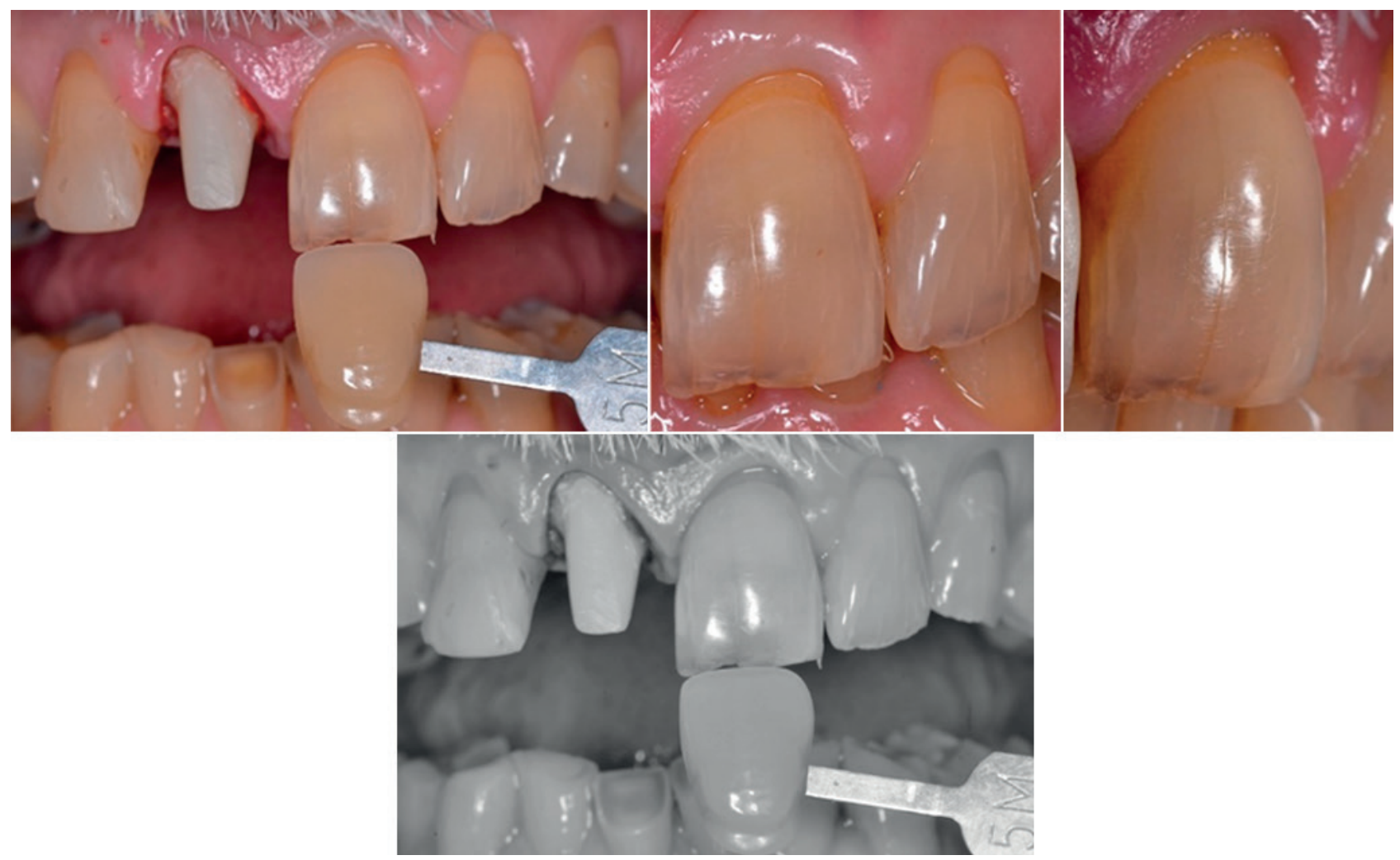

Figura 5. A. Set fotográfico de pieza homóloga al laboratorio dental. B. Fotografía en escala de grises

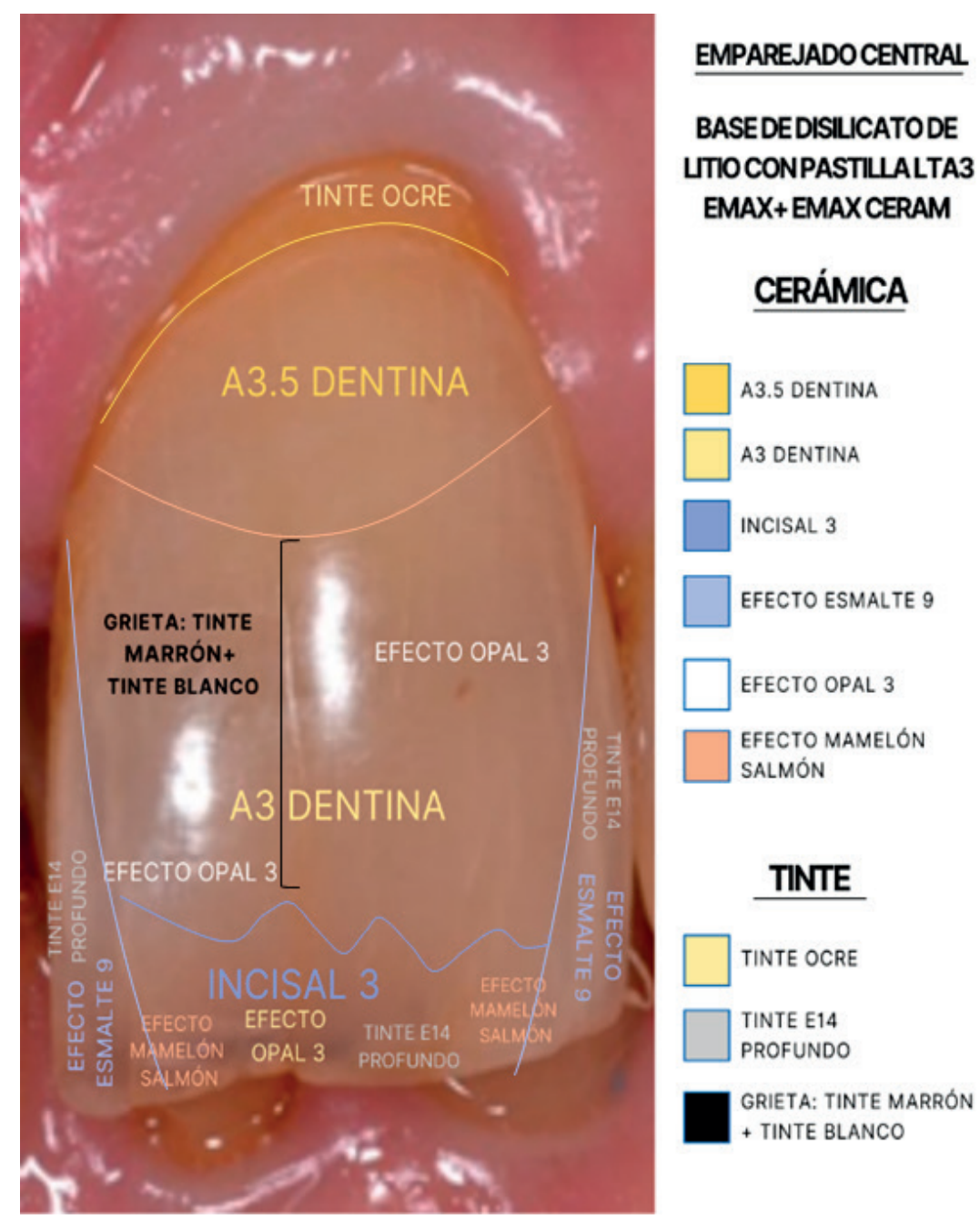

Figura 6. Mapa cromático y guía de caracterizaciones 
E-max press, HT A 3 (Ivoclar-Vivadent, Pforzheim, Alemania) usando la técnica de estratificación y maquillado con IPS E-max Ceram; para un mejor terminado y camuflaje se empleó el Kit Ivocolor de la misma casa comercial para emular las características del diente homólogo, logrando una mimetización de alto nivel (Figura 7).

Cuarta consulta: en la cita de cementación, tras remover el provisorio y limpiar adecuadamente el remanente dental se realizó la prueba de color, forma, ajuste marginal y control oclusal; a la total aprobación del paciente y odontólogo, se decidió proceder a la cementación de la corona.

El protocolo de cementación consistió en la preparación interna de la corona de disilicato de litio mediante grabado con gel de ácido fluorhídrico al 9\% (porcelain etch, Ultradent, Utah, USA) por 20 segundos, lavado por 1 minuto, secado, silanización por 1 minuto (silano, Ultradent, Utah, USA) y colocación de adhesivo sin fotoactivar (Scotchbond Universal, 3M, Minnesota, USA); bajo aislamiento relativo se acondiciona la estructura dental usando ácido ortofosfórico al 35\% (Ultra-Etch, Ultradent, Utah, USA) por 15 segundos, lavado, secado y colocación de adhesivo (Scotchbond universal 3M, Minnesota, USA) fotoactivándolo por 20 segundos. Para la cementación se aplicó cemento resinoso color A2 (AllCem Core, FGM, Joinville, Brasil), retirando los excesos circundantes con un pincel y completando la fotopolimerización (Elipar-DeepCure-L, 3M, Minnesota, USA) con 60 segundos de luz por cada lado; para finalizar se limpió cualquier excedente interproximal usando seda dental y lijas de poliéster.

Resultados del tratamiento. Tras la correcta aplicación de los protocolos clínicos, se logró satisfacer las expectativas del paciente mediante una corona de disilicato de litio, mostrando naturalidad en relación a las piezas naturales adyacentes (Figura 8).

\section{Discusión}

La luz ideal para la toma de color será aquella más próxima al espectro de la luz solar diurna recomendándose fuentes de luz corregidas, que ofrecen temperaturas de color de 5000 a $6500{ }^{\circ} \mathrm{K}^{3}$. También es interesante la observación bajo dos fuentes de luz diferentes (luz natural y luz artificial), con el fin de asegurar todavía más la selección, ya que en ocasiones dos objetos (guía de color y diente) pueden verse del mismo color bajo una fuente de luz y de diferente color bajo otra, este fenómeno se denomina metamerismo ${ }^{3}$.

Aunque no existe una guía de colores que reproduzca todas las tonalidades, la escala de color Vita 3D master establece grupos por su luminosidad, saturación cromática creciente y tono, lo cual otorga mayor coincidencia; esta escala permite medir 2 parámetros importantes: el matiz y el valor de un objeto determinado ${ }^{4}$.

El uso de registro fotográfico favorece la documentación, el diagnóstico y el plan de tratamiento, contiene más detalles que cualquier explicación escrita o dibujada, cuánta más información tenga el técnico, más similitud y naturalidad tendrá el diente en el resultado final. El clínico debe familiarizarse con las aplicaciones fotográficas, las técnicas y los diversos equipos y programas que existen en la actualidad y transformarlos en una herramienta optima dentro de la práctica cotidiana ${ }^{14,15}$.

La elaboración del mapa cromático, facilita la elección simplificada y rápida de los materiales restauradores, estas anotaciones expresan el color predominante y las zonas en las que este puede variar comenzando desde las áreas cervicales hasta las incisales donde en ocasiones se

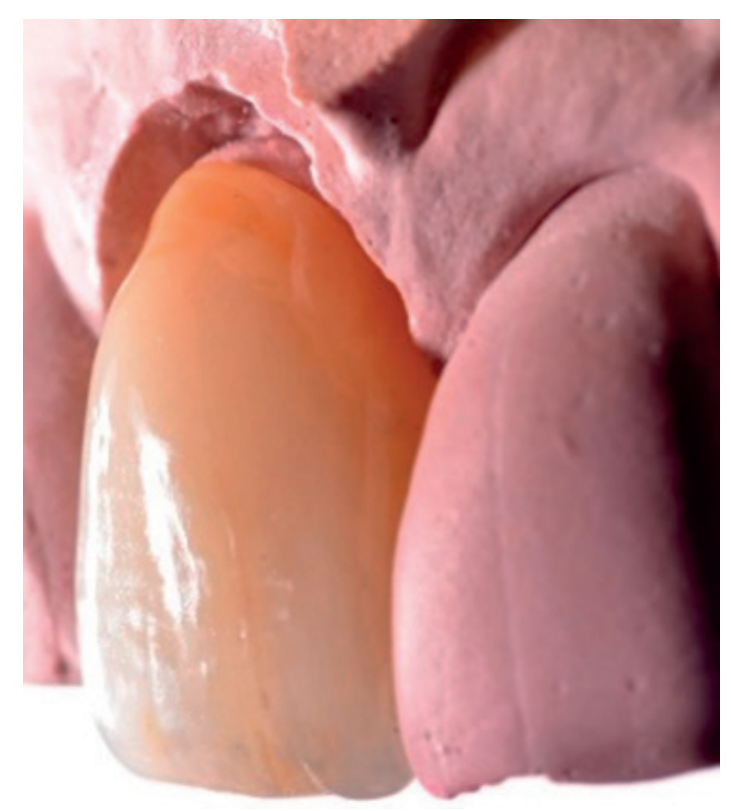

Figura 7. Corona de disilicato de lítio IPS E-max press 


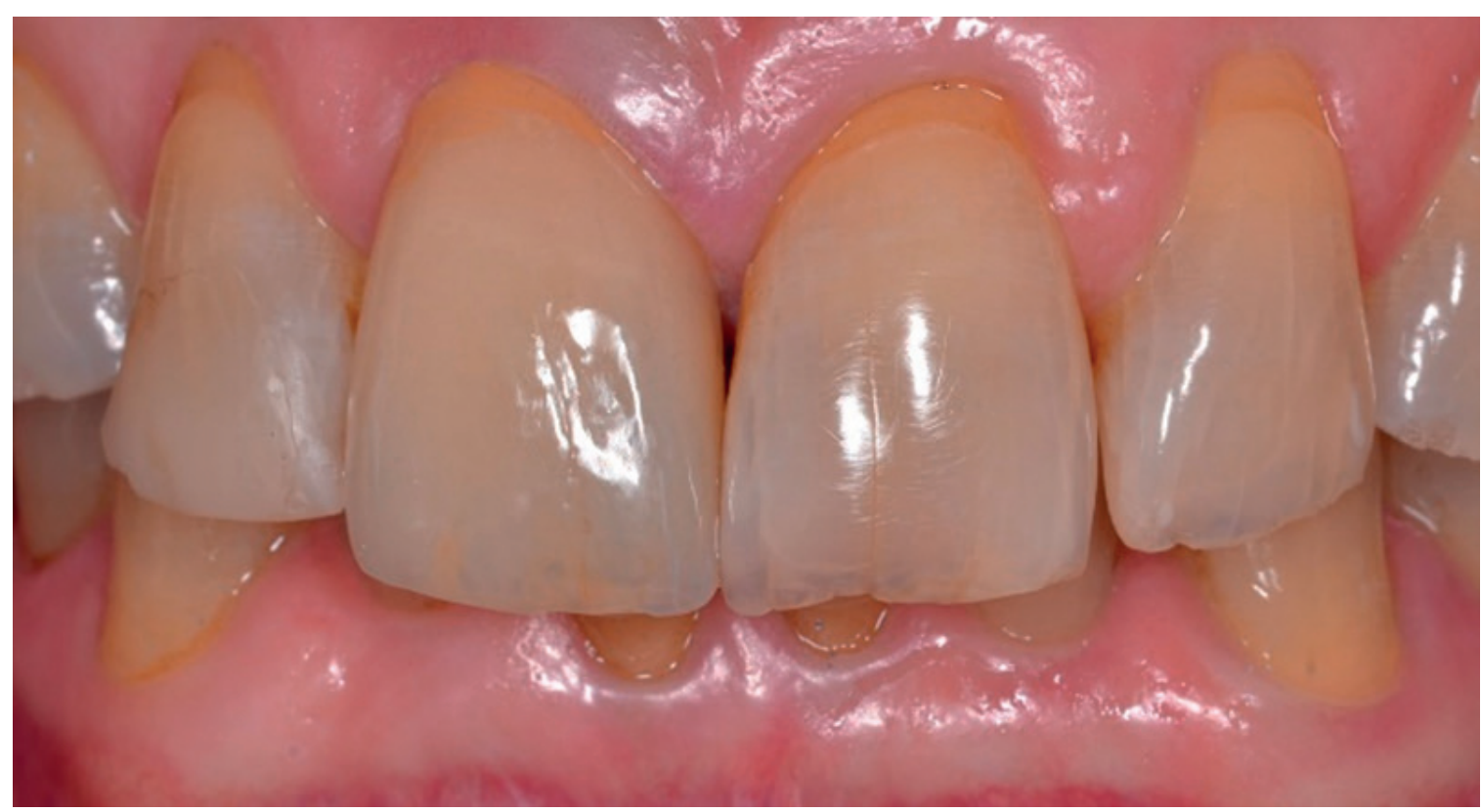

Figura 8. Aspecto final, tras cementación y ajustes oclusales

requiere del uso de fondos coloridos como rojo, azul o negro contrastando mejor las variaciones de esmalte y dentina, así como de las demás características dentales. Cuando se usan aparatos electrónicos los mapas cromáticos son más detallados y precisos, algunos incluso permiten la personalización de las guías combinándolo con particularidades de ciertos materiales restauradores ${ }^{16,17}$.

Tras la digitalización de los medios de comunicación y el uso frecuente de aplicaciones como WhatsApp o los correos electrónicos ha evolucionado la relación entre el clínico y el laboratorio dental, el intercambio de datos es cada vez más fluido, didáctico y en algunas ocasiones en tiempo real, facilitando y agilizando los resultados que ante las exigencias de los pacientes necesitan conjugar esfuerzos, conocimientos para lograr un tratamiento exitoso ${ }^{18}$.

Se concluye que, para el correcto manejo clínico de casos de alta demanda estética es primordial una comunicación acertada entre el clínico y el técnico protésico dental, por medio de mapas cromáticos, selección adecuada de color y documentación fotográfica de alta calidad; con lo cual se logra la elaboración de elementos protésicos que puedan satisfacer las demandas estéticas del paciente, logrando simular las características propias de una pieza dental.

\section{Agradecimiento}

Se agradece al laboratorio dental "Dentales MM" y al ceramista Marco Antonio Méndez Méndez por su invaluable colaboración en la elaboración del presente reporte.

\section{Referencias bibliográficas}

1. Torrecilla Venegas R, Castro Gutiérrez I. Efectos del envejecimiento en la cavidad bucal. Revista 16 de Abril [Internet]. 2020 [citado el 18 de mayo de 2021];
50(278):819. Disponible en: http://www.rev16deabril. sld.cu/index.php/16_4/article/view/819.

2. Mujica Sánchez N. La importancia de la selección del color en la práctica Odontológica. Psychol lat [Internet]. 2018 [citado el 14 de marzo de 2021]; spec:330333. Disponible en: https://psicologia.ucm.es/data/ cont/docs/29-2019-02-15-Mujica\%20S\%C3\%A1nchez\%20-.pdf.

3. González Sandoval J, Martínez Alvarez MS, Brand Rubalcava A, Torres Ramos, Román Godínez, Mendizabal Ruiz. Mapeo crómatico dental a partir de imagenes digitales. ReCIBE [Internet]. 2015 [citado el 14 de marzo de 2021]; 4(3):1-22. Disponible en: https://www. http://recibe.cucei.udg.mx/index.php/ReCIBE/article/ view/45/43.

4. Bersezio C, Oliveira Jr OB, Vildósola P, Martín J, Fernández $\mathrm{E}$, Angel $\mathrm{P}$, et al. Instrumentación para el registro del color en odontología. Rev dent Chile (Santiago) [Internet]. 2013 [citado el 16 de marzo de 2021]; 104(3):3-7. Disponible en: https://www.researchgate. net/publication/262065490_Instrumentacion_para_el_ registro_del_color_en_odontologia_-_Review.

5. Schmeling M. Selección del color y reproducción en Odontología. Parte3: Escogencia del color de forma visual e intrumental. Odovtos-Int J Dent Sc. 2017;19(1):2332. DOI: http://dx.doi.org/10.15517/ijds.v0i0.28083.

6. Taberner A. Medidas para la mejora de la comunicación entre el laboratorio y la clinica dental. Gaceta Dental [Internet]. 2013 [citado el 19 de marzo de 2021]; 24(245):168-183. Disponible en: https://gacetadental. com/2013/03/medidas-para-la-mejora-de-la-comunicacion-entre-el-laboratorio-y-la-clinica-dental-24020/

7. Carrillo Baracaldo JS, Álvarez Quesada C, Calatayud Sierra J, Fernández Sánchez J. Trabajo en equipo en Odontología: la comunicación con el laboratorio dental como clave de éxitos clínico. A propósito de un caso clínico de tratamiento multidisciplinar. Cient Dent. 2006;3(2):129-36. 
8. Figueroa RI, Cruz FG, De Carvalho RF, Leite FPP, Chaves MAM. Rehabilitación de los dientes anteriores con el sistema cerámico Disilicato de litio. Int J Odontostomat. 2014;8(3):469-74. DOI: http://dx.doi.org/10.4067/ S0718-381X2014000300023.

9. Díaz Azueta NM, Flores Díaz RA, García Contreras R. Rehabilitación del sector anterosuperior mediante coronas libres de metal de disilicato de litio. reporte de un caso. Rev Odontol latinoam [Internet]. 2017 [citado el 21 de marzo de 2021]; 9(1):29-33. Disponible en: https://www.odontologia.uady.mx/revistas/rol/pdf/ V09N1p29.pdf.

10. Martínez Galeano G, Pacheco Muñóz L, López Palomar LC. Selection of dental ceramics in an esthetic area. A case report. Rev Fac Odontol Univ Antioq. 2017;29(1):222-40. DOI: http: //dx.doi.org/10.17533/ udea.rfo.v29n1a12.

11. Bravo A, Villarreal M, Paredes M. Una mirada acerca de restauraciones cerámicas. Dom Cien. 2019;5(1):24778818. DOI: http://dx.doi.org/10.23857/dc.v5i1.866.

12. McLean A. Criteria for the predictably restorable endodontically treated tooth. J Can Dent Ass [Internet]. 1998 [citado el 18 de mayo de 2021]; 64:782-787. Disponible en: http://cda-adc.ca/jcda/vol-64/issue-11/mclean-e.pdf.

13. Stockton LW. Factors affecting retention of post system: A literature review. J Prosthet Dent. 1999;81(4):380-5. DOI: 10.1016/s0022-3913(99)80002-x.
14. González Pérez JC, Cedeño Díaz Leal Y, Sánchez Barrios V, Rivera Martínez G, Santiago Chávez L. La importancia de la fotografía clínica en la odontología. Arch Inv Mat Inf [Internet]. 2019 [citado el 22 de marzo del 2021]; 10:88-90. Disponible en: https://www.medigraphic.com/pdfs/imi/imi-2019/imi193b.pdf.

15. Moscardó P, Camps Alemany I. Odontología estética: Apreciación cromática en la clínica y el laboratorio. Med Oral Patol Oral Cir Bucal [Internet]. 2006 [citado el 29 de marzo de 2021]; 11(4):363-368. Disponible en: https://scielo.isciii.es/scielo.php?script=sci_abstract\&pid $=$ S1698-69462006000400015.

16. Castro Rivas K, Concha Urgiles G. Importancia del mapeo cromático dental en el resultado estético de restauraciones directas en el sector anterior. [tesis de licenciatura]. [Guayaquil]:Facultad de Odontología, Universidad de Guayaquil; 2019.

17. Loayza Paliz SV, Andrade M. Toma de color dental mediante el método visual y el espectrofotómetro. [tesis de licenciatura]. [Guayaquil]: Facultad de Odontología, Universidad de Guayaquil; 2020.

18. Gaceta Dental. La comunicación entre el clínico y el laboratorio debe ser cada vez más estrecha. [Consultado el 17 de abril 2021]. Accesible en: https:// gacetadental.com/2016/09/la-comunicacion-entre-el-clinico-y-el-laboratorio-debe-ser-cada-vez-mas-estrecha-60898/ 